\title{
Guidelines Translation for Distress Management in Cancer Patients Based on CAN-IMPLEMENT: Adaptation Part
}

\author{
Liang Fu \\ Fudan University/Affiliated Jinhua Hospital, Zhejiang University School of Medicine \\ Yang Yang \\ Fudan University Shanghai Cancer Center \\ Yan Hu ( $\nabla$ huyan@fudan.edu.cn) \\ Fudan University https://orcid.org/0000-0001-6506-0694 \\ Zhenqi Lu ( $\square$ luzhenqi1972@163.com ) \\ Fudan University Shanghai Cancer Center \\ Xiaoju Zhang \\ Fudan University Shanghai Cancer Center \\ Mingzhu Huang \\ Fudan University Shanghai Cancer Center \\ Yuanyuan Li \\ Zhongshan Hospital Affiliated to Fudan University \\ Fuzhong Zhu \\ Fudan University Shanghai Cancer Center \\ Yang Wang \\ Fudan University Shanghai Cancer Center \\ Zhe Huang \\ Fudan University Shanghai Cancer Center
}

\section{Research}

Keywords: Clinical Practice Guidelines, Translation, Adaptation, Cancer, Distress Management

Posted Date: August 17th, 2020

DOI: https://doi.org/10.21203/rs.3.rs-53486/v1

License: 두 (i) This work is licensed under a Creative Commons Attribution 4.0 International License. Read Full License

Version of Record: A version of this preprint was published at International Journal of Nursing Sciences on December 16th, 2021. See the published version at https://doi.org/10.1016/j.ijnss.2021.12.005. 


\section{Abstract \\ Background}

Almost all cancer patients experience some level of distress, which has significantly reduced their quality of life. Nowadays evidencebased practice in various fields of health care is increasing, and it has played an important role. This study aimed to clarify the theme of guideline adaptation, integrate relevant clinical practice guidelines on distress management in cancer patients, and develop the Cancer-related Distress Management Guidelines.

\section{Methods}

A Guideline Adaptation and Implementation Planning Resource (CAN-IMPLEMENT) was used as the research framework, which include three phases. Here the Adaptation Part (Phase 1) was reported: adaptation of relevant clinical practice guidelines on the distress management in cancer patients to form the Cancer-related Distress Management Guidelines.

\section{Results}

The physical symptom distress score $(0.61 \pm 0.58)$ was almost equal to the psychological symptom distress score $(0.61 \pm 0.70)$ among cancer patients in Shanghai. A work plan for the adaptation of Cancer-related Distress Management Guidelines was developed. Two clinical practice guidelines related to distress management in cancer patents were included after searching, screening, assessment and selection. The domain scores of the draft the Cancer-related Distress Management Guidelines on Appraisal of Guidelines for Research and Evaluation II (AGREE II) were 80.00\% (scope and purpose), 82.78\% (stakeholder involvement), 87.50\% (rigour of development), 73.89\% (clarity and presentation), 73.75\% (applicability), $87.50 \%$ (editorial independence) respectively. The scores of most recommendations on feasibility, appropriateness, meaningfulness and effectiveness were $90.0 \%$ or higher. The final guidelines included 13 recommendations.

\section{Conclusions}

The psychological symptoms and physical symptoms were both worthy of attention in cancer patients. The quality of the draft the Cancer-related Distress Management Guidelines was well-accepted based on two included guidelines. Most recommendations received positive and constructive feedback on feasibility, appropriateness, meaningfulness and effectiveness. The final guidelines was formed based on the revision of the first draft guidelines.

\section{Contributions To The Literature}

- A survey on the current situation can be a highly effective approach to identify guidelines adaptation theme, and provide objective background information on a certain problem in the research site.

- This guidelines adaptation only included de novo clinical practice guidelines, which greatly reduce the workload, decrease the complexity and improve the operability. Thus, the variability of included guidelines can be decreased, and the quality of current adapted guidelines can be guaranteed further.

- The guidelines adaptation was feasible in low and middle income countries based on CAN-IMPLEMENT and guided by the Knowledge-to-Action Framework.

\section{Background}

With the progress of cancer treatment, the survival time of cancer patients is prolonged [1,2]. The prolongation of survival time places higher demands on existing health care services, with a view to better improving the quality of life (QoL) in cancer patients. It is well known that cancer patients always have a series of physiological symptoms and/or psychological symptoms due to the disease and/or related treatment, such as distress, sleep disturbance, fatigue, lack of appetite and so on [3,4,5]. A literature review revealed that the symptom burden of cancer patients has a great negative impact on the patients' QoL $[6,7,8]$. At the beginning of the study, the current results were inconsistent on frequency, severity, distress of physical and psychological symptoms in different studies $[3,4,9]$. In 
view of the large scope of symptom management for cancer patients, it was not easy on determining the research topic. As a result, a cross-sectional survey was conducted to clarify the status of symptom burden among cancer patients in the research site [10]. The Memorial Symptom Assessment Scale Short Form (MSAS-SF) was chosen as the instrument in the investigation, because its items are comprehensive (covering both physical and psychological symptoms) and convenient (only the distress or frequency of symptoms is evaluated), which is easy for cancer patients to fill in [10,11]. Finally, distress was identified as the research theme based on the investigation, literature review and professional judgment. It is reported that the positive rate and severity of distress were both high in cancer patients [12,13], which significantly reduced their quality of life [14]. Distress management can clarify the patient's distress status, understand their experience of distress, and effectively alleviate their distress through relevant management strategies [12, 15 , 16]. Thus, it is necessary to carry out distress management in cancer patients under the guidance of appropriate theories, models, frameworks, methods and so on.

Nowadays there is increasing understanding and implementing evidence-based practice in various fields of health care, and it has played an important role in regulating clinical practice, improving patient outcomes, optimizing resource allocation and so on. To date, many evidence-based practice models, frameworks and methods have been launched by different organizations worldwide. For example, the JBI Model of Evidence-based Healthcare developed by the Joanna Briggs Institute [17, 18], the Ottawa Model of Research Use reported by Logan and Graham [19], the Promoting Action on Research Implementation in Health Service Framework published by Kitson et al. [20, 21, 22], the Knowledge-to-Action Framework by Graham et al. [23], and A Guideline Adaptation and Implementation Planning Resource (CAN-IMPLEMENT) presented by Harrison and her colleagues [24, 25]. Different models, frameworks and methods are suitable for different situations, and healthcare professionals can choose the appropriate model, framework or method according to the actual situation. CAN-IMPLEMENT is guided by the Knowledge-to-Action Framework, which also includes two parts of knowledge creation and action cycle. The action cycle part is divided into three stages: identification and clarification of the practice issue, solution building, implementation, evaluation and sustainability [24], aiming to facilitate the translation of existing guidelines into clinical practice by adaptation, alignment, application and evaluation. Therefore, CAN-IMPLEMENT is different from other evidence-based practice models, frameworks, and methods, which emphasizes the utilization of the primary evidence resource of clinical practice guidelines (including systematic reviews), rather than all types of complex evidence resources. This helps to simplify the process of evidence synthesis, and facilitates clinical practitioners to master the method, thereby promoting the clinical translation of guidelines more conveniently and quickly.

\section{Aim}

The entire study aimed to carry out guidelines adaptation and implementation for distress management in cancer patients, which was based on CAN-IMPLEMENT and guided by the Knowledge-to-Action Framework. This section focused on adaptation process. The implementation part was reported in another paper. The Adaptation Part (Phase 1) was to clarify the theme of guideline adaptation, integrate relevant clinical practice guidelines on distress management in cancer patients, and develop the Cancer-related Distress Management Guidelines. The specific objectives in this were as follows: (1) To investigate the status of related symptoms in cancer patients; (2) To search and screen existing relevant clinical practice guidelines on distress management in cancer patients systematically and comprehensively; (3) To assess and select relevant clinical practice guidelines on distress management in cancer patients rigidly and normatively; (4) To draft the Cancer-related Distress Management Guidelines by selecting, extracting and integrating the included clinical practice guidelines on distress management in cancer patients; (5) To revise and endorse the final Cancer-related Distress Management Guidelines by peer review.

\section{Methods}

Ethics approval for this study was obtained from School of Nursing XX University (IRB\#2017-9-3) and XX University XX Cancer Centre (1710177-23). From May 2017 to June 2019, the study 'Guidelines Adaptation and Implementation for Distress Management in Cancer Patients' was conducted in School of Nursing, XX University and three medical oncology units, XX Cancer Center, China. The main contents of the above study were translating relevant guidelines on distress management into clinical practice, which was based on CAN-IMPLEMENT and guided by the Knowledge-to-Action Framework. The translation and application of CAN-IMPLEMENT in China was authorized by the principal investigator of CAN-IMPLEMENT project.

According to CAN-IMPLEMENT, this study consisted of three phases: (1) integrating relevant clinical practice guidelines on the distress management in cancer patients to form the Cancer-related Distress Management Guidelines; (2) aligning the Cancer-related Distress 
Management Guidelines into clinical practice among gastric cancer patients receiving chemotherapy; (3) monitoring, evaluating and maintaining the implementation of the Cancer-related Distress Management Guidelines in clinical practice. Here the Adaptation Part (Phase 1) was reported.

\title{
Phase 1 Identification and Clarification of the Practice Issue: Guidelines Adaptation
}

Step 1 Call to Action: To clarify the theme of this guidelines adaptation, a cross-sectional study was carried out from September 25, 2017 to December 28, 2017. The Simplified Chinese Version of the Memorial Symptom Assessment Scale Short Form (MSAS-SF-SC) was handed out to 384 adult cancer patients in three medical oncology units of XX University XX Cancer Center.

Step 2 Guideline Development Plan: The health problem of this guidelines adaptation was articulated based on PIPOH (P-Population, IIntervention, P-Professionals/Patients, O-Outcomes, H-Health Care Setting). The feasibility was confirmed through preliminary search for existing guidelines on distress management. A multidisciplinary panel of guidelines adaptation was set up to manage this guidelines adaptation. The consensus process and the work plan was determined by the guidelines adaptation panel using the expert meeting method.

Step 3 Search and Screen: Three researchers (a professor skilled with evidence-based practice methodologies, a doctoral candidate and a postgraduate student of nursing science) searched and screened all guidelines on distress management for cancer patients using the literature research method, which published on guidelines website, professional website and medical database from 1 st April 2013 to 31st March 2018. Additional references were identified through an examination of the references from included guidelines.

Step 4 Assess and Select: Four appraisers (a doctoral candidate and three postgraduate students of nursing science) evaluated guidelines using the literature evaluation method with Appraisal of Guidelines for Research and Evaluation II (AGREE II). A professor and an associate professor skilled with evidence-based practice methodologies were involved if there were disagreements among the four appraisers. The included guidelines were selected by the guidelines adaptation panel. Only de novo clinical practice guidelines were included in this guidelines adaptation.

Step 5 Draft, Revise and Endorse: The draft adapted guidelines were written based on guidelines adaptation method by the guidelines adaptation panel. The fundamental principles of this guidelines adaptation were as follow: (1) the recommendations in different guidelines are consistent, then the original recommendations are accepted; (2) the recommendations in different guidelines are not consistent or the recommendations are not clear, then certain original recommendations are accepted or new adapted recommendations are formed by comparing and analyzing the original guidelines' evidence descriptions, evidence sources, quality of evidence, strength of recommendation and so on, combined with professional judgments.

Ten stakeholder representatives (an associate professor skilled with evidence-based practice methodologies, a chief physician of psychological medicine, a chief physician of palliative care, a chief physician of clinical epidemiology, a professor and a supervisor nurse of oncology nursing, an associate physician of oncology, an associate professor of psychological care, a social worker, a breast cancer patient and her husband) were invited to review the draft adapted guidelines on June 11, 2018. The on-site expert review method was adopted with AGREE II and self-designed recommendations review form (Based on The JBI Model of Evidence-based Healthcare) $[17,18]$. Additional file 1 . The final guidelines were revised and formed by the guidelines adaptation panel, and a renewal plan was established.

\section{Results}

\section{Phase 1 Identify Issue/problem: Guidelines Adaptation}

\author{
Step 1 Call to Action
}

The top ten symptoms of cancer patients were lack of energy (53.8\%), dry mouth (44.0\%), numbness/tingling in hands and feet (39.3\%), feeling irritable (38.2\%), hair loss (36.5\%), pain (35.7\%), worrying (35.1\%), difficulty sleeping (34.0\%), lack of appetite (33.4\%), and feeling sad (31.2\%). The distress score of physical symptom $(0.61 \pm 0.58)$ was almost equal to the distress score of psychological

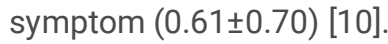

Step 2 Guideline Development Plan 
The health problem was P (cancer patients), I (screening, assessment, care, referral and treatment), P (professionals and patients), 0 (distress), $\mathrm{H}$ (tumor hospital, oncology department and so on). Several related guidelines were found in the preliminary search, such as the Distress Management Guidelines of National Comprehensive Cancer Network (NCCN) [13], A Pan Canadian Practice Guideline: Screening, Assessment and Care of Psychosocial Distress, Depression, and Anxiety in Adults with Cancer [26]. The guidelines adaptation panel consisted of a professor and two associate professors skilled with evidence-based practice methodologies, a chief physician and an associate chief physician of oncology, a professor, two associate professors, four supervisor nurses and a nurse practitioner of oncology nursing, an associate professor of psychological care, an attending doctor of psychological medicine, a social worker, a doctoral candidate and a postgraduate student of nursing science. The determined consensus methods were consensus conference and voting.

A work plan for the adaptation of the Cancer-related Distress Management Guidelines was written, including background, the health problem, the multidisciplinary panel, consensus methods, evidence sources, methodology for reporting clinical practice guidelines, methods of guidelines adaptation, conflicts of interest statement and so on.

Step 3 Search and Screen Guidelines

The searching initially retrieved 1864 references from guidelines websites $(n=105)$, professional agency websites $(n=145)$ and medical database $(n=1614)$. Additional file 2 . Other 8 references were identified by tracing the references of including references. After exclusion of 725 duplicates, 1147 were screened by title and abstract. Following title and abstract screening, 138 references continued on to full-text screening. Finally, two clinical practice guidelines related to distress management in cancer patients were found after searching and screening. Figure 1. The two obtained guidelines were Distress Management [13] and Chinese Psychosocial Oncology Therapy Guidelines for Cancer Patients [27].

Step 4 Assess and Select Guidelines

Distress Management were developed by Jimmie C. Holland's team, and published by NCCN on February 23, 2018. Another obtained guidelines were Chinese Psychosocial Oncology Therapy Guidelines for Cancer Patients, developed by Lili Tang, et al and published by China Anti-Cancer Association on June 18, 2016. The scores of each AGREE II domain were $54.17 \%-81.94 \%$ and $44.27 \%-88.89 \%$ respectively. The scores of overall quality of the two obtained guidelines were 3 to 6 . Table 1 . The guidelines adaptation panel decided to include both guidelines based on the results of assessment with consensus.

Table 1

The scores and overall assessment of AGREE II for the included guidelines

\begin{tabular}{|c|c|c|c|c|c|c|c|c|c|c|}
\hline \multirow[t]{2}{*}{ Titles } & \multirow{2}{*}{$\begin{array}{l}\text { Scope } \\
\text { and } \\
\text { Purpose }\end{array}$} & \multirow{2}{*}{$\begin{array}{l}\text { Stakeholder } \\
\text { Involvement }\end{array}$} & \multirow{2}{*}{$\begin{array}{l}\text { Rigour of } \\
\text { Development }\end{array}$} & \multirow{2}{*}{$\begin{array}{l}\text { Clarity and } \\
\text { Presentation }\end{array}$} & \multirow[t]{2}{*}{ Applicability } & \multirow{2}{*}{$\begin{array}{l}\text { Editorial } \\
\text { Independence }\end{array}$} & \multirow{2}{*}{$\begin{array}{l}\text { Overall } \\
\text { Quality }\end{array}$} & \multicolumn{3}{|c|}{ Recommendations } \\
\hline & & & & & & & & $\mathrm{Y}$ & YM & $\mathbf{N}$ \\
\hline A & $81.94 \%$ & $75.00 \%$ & $56.25 \%$ & $54.17 \%$ & $59.38 \%$ & $70.83 \%$ & $\begin{array}{l}6,3,4 \\
6\end{array}$ & 1 & 3 & 0 \\
\hline B & $88.89 \%$ & $59.72 \%$ & $44.27 \%$ & $86.11 \%$ & $53.13 \%$ & $87.50 \%$ & $\begin{array}{l}6,5,6 \\
4\end{array}$ & 3 & 1 & 0 \\
\hline
\end{tabular}

Step 5 Draft, Revise and Endorse

The first draft Cancer-related Distress Management Guidelines consisted of background, evidence, method, process, recommendations, other aspects, references and attachments and so on. There were 14 recommendations in the first draft, which involving screening, assessment, care, referral, treatment and so on. Table 2. The scores of AGREE II domains were $80.00 \%$ (scope and purpose), $82.78 \%$ (stakeholder involvement), $87.50 \%$ (rigour of development), 73.89\% (clarity and presentation), $73.75 \%$ (applicability), and $87.50 \%$ (editorial independence). The scores of overall quality were $6,6,6,6,6,6,4,6,6$ and 6 . All appraisers would recommend this guidelines, and only one of them would recommend with modifications. Table 3 . The scores of most recommendations on feasibility, appropriateness, meaningfulness and effectiveness were $90.0 \%$ or higher. Table 4. 
The recommendations in the draft Distress Management Guidelines in Cancer Patients

\begin{tabular}{|c|c|}
\hline Number & Recommendations \\
\hline 1 & $\begin{array}{l}\text { All patients should be assessed prior to clinical visits using the Distress Thermometer, and immediate screenings should } \\
\text { be provided during the vulnerable period. }\end{array}$ \\
\hline 2 & $\begin{array}{l}\text { If the patient's distress is mild (thermometer score }<4) \text {, the primary oncology team should assess the possible distress } \\
\text { related symptoms of the patient. }\end{array}$ \\
\hline 3 & $\begin{array}{l}\text { According to the assessment results of possible distress related symptoms, the primary oncology team manages the } \\
\text { symptoms associated with the patient's distress. }\end{array}$ \\
\hline 4 & $\begin{array}{l}\text { After the primary oncology team manages the symptoms associated with the patient's distress, the Distress } \\
\text { Thermometer is used again for follow-up screening. }\end{array}$ \\
\hline 5 & $\begin{array}{l}\text { If the patient's distress is moderate or severe (thermometer score } \geq 4 \text { ), further clinical evaluations should be provided by } \\
\text { oncology team, including clinical interviews, anxiety/depression assessment, etc. }\end{array}$ \\
\hline 6 & $\begin{array}{l}\text { According to the results of further clinical evaluations, it is recommended to refer patients to mental health } \\
\text { professionals, social workers, chaplains, medical staff for further diagnosis and treatment. }\end{array}$ \\
\hline 7 & Mental health professionals provide psychoeducation for patients in need. \\
\hline 8 & Mental health professionals provide cognitive behavioral therapy for patients in need. \\
\hline 9 & Mental health professionals provide supportive psychotherapy for patients in need. \\
\hline 10 & Mental health professionals provide family and couples therapy for patients in need. \\
\hline 11 & Social workers provide support and counseling to patients in need. \\
\hline 12 & Chaplains provide spiritual and chaplaincy care for patients in need. \\
\hline 13 & Medical staff provide palliative care for patients in need. \\
\hline 14 & $\begin{array}{l}\text { Patients who were referred or not referred to mental health professionals, social workers, chaplains, medical staff were } \\
\text { further screened using the Distress Thermometer. }\end{array}$ \\
\hline
\end{tabular}

Table 3

The scores and overall assessment of AGREE II for the adapted guidelines

\begin{tabular}{|c|c|c|c|c|c|c|c|c|c|c|}
\hline \multirow[t]{2}{*}{ Titles } & \multirow{2}{*}{$\begin{array}{l}\text { Scope } \\
\text { and } \\
\text { Purpose }\end{array}$} & \multirow{2}{*}{$\begin{array}{l}\text { Stakeholder } \\
\text { Involvement }\end{array}$} & \multirow{2}{*}{$\begin{array}{l}\text { Rigour of } \\
\text { Development }\end{array}$} & \multirow{2}{*}{$\begin{array}{l}\text { Clarity and } \\
\text { Presentation }\end{array}$} & \multirow[t]{2}{*}{ Applicability } & \multirow{2}{*}{$\begin{array}{l}\text { Editorial } \\
\text { Independence }\end{array}$} & \multirow{2}{*}{$\begin{array}{l}\text { Overall } \\
\text { Quality }\end{array}$} & \multicolumn{3}{|c|}{ Recommendation } \\
\hline & & & & & & & & $Y$ & YM & $\mathbf{N}$ \\
\hline C & $80.00 \%$ & $82.78 \%$ & $87.50 \%$ & $73.89 \%$ & $73.75 \%$ & $87.50 \%$ & $\begin{array}{l}6,6,6 \\
6,6,6 \\
4,6,6 \\
6\end{array}$ & 9 & 1 & 0 \\
\hline
\end{tabular}

C: Distress Management Guidelines in Cancer Patients; Y: Yes; YM: Yes, with modifications; N: No. 
Table 4

The scores on feasibility, appropriateness, meaningfulness and effectiveness of recommendations in the adapted guidelines $(n=10)$

\begin{tabular}{|c|c|c|c|c|c|c|c|c|}
\hline \multirow[t]{2}{*}{ Recommendations } & \multicolumn{2}{|c|}{ Feasibility } & \multicolumn{2}{|c|}{ Appropriateness } & \multicolumn{2}{|c|}{ Meaningfulness } & \multicolumn{2}{|c|}{ Effectiveness } \\
\hline & $\mathrm{n}$ & $\%$ & $\mathrm{n}$ & $\%$ & $\mathbf{n}$ & $\%$ & $\mathbf{n}$ & $\%$ \\
\hline 1 & 10 & 100.0 & 10 & 100.0 & 9 & 90.0 & 9 & 90.0 \\
\hline 2 & 10 & 100.0 & 9 & 90.0 & 9 & 90.0 & 10 & 100.0 \\
\hline 3 & 10 & 100.0 & 10 & 100.0 & 9 & 90.0 & 9 & 90.0 \\
\hline 4 & 10 & 100.0 & 9 & 90.0 & 10 & 100.0 & 10 & 100.0 \\
\hline 5 & 9 & 90.0 & 10 & 100.0 & 10 & 100.0 & 10 & 100.0 \\
\hline 6 & 6 & 60.0 & 9 & 90.0 & 9 & 90.0 & 9 & 90.0 \\
\hline 7 & 8 & 80.0 & 9 & 90.0 & 9 & 90.0 & 9 & 90.0 \\
\hline 8 & 9 & 90.0 & 9 & 90.0 & 10 & 100.0 & 10 & 100.0 \\
\hline 9 & 10 & 100.0 & 9 & 90.0 & 10 & 100.0 & 10 & 100.0 \\
\hline 10 & 10 & 100.0 & 10 & 100.0 & 10 & 100.0 & 10 & 100.0 \\
\hline 11 & 10 & 100.0 & 10 & 100.0 & 10 & 100.0 & 10 & 100.0 \\
\hline 12 & 9 & 90.0 & 10 & 100.0 & 10 & 100.0 & 10 & 100.0 \\
\hline 13 & 4 & 40.0 & 6 & 60.0 & 7 & 70.0 & 7 & 70.0 \\
\hline 14 & 10 & 100.0 & 9 & 90.0 & 10 & 100.0 & 10 & 100.0 \\
\hline
\end{tabular}

For the final guidelines, the main contents were the same with the draft guidelines, but revisions were made based on the recommendations from stakeholder representatives. Firstly, Chinese word "眈四" was adopted as the formal translation for "distress",

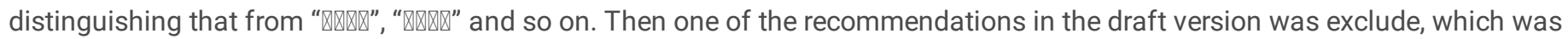
"According to the results of further clinical evaluations, it is recommended to refer patients to mental health professionals, social workers, life meaning support staff, medical staff, etc. for further diagnosis and treatment". Therefore, only 13 recommendations were included in the final version. In addition, it is not recommended to use "spiritual and chaplaincy care", which was replaced by "life meaning care". The Cancer-related Distress Management Guidelines will be updated in five years using the same methods in this paper.

\section{Discussion}

The symptom burden of cancer patients in Shanghai was high. The distress of physical symptoms and psychological symptoms were almost equivalent [10]. Therefore, psychological symptoms and physical symptoms were both worthy of attention. Distress is a multifactorial unpleasant experience of a psychological (ie, cognitive, behavioral, emotional), social, spiritual, and/or physical nature [13], which includes both physical and psychological symptoms, and coincides with the results of this study. Meanwhile, the definition of distress is clear, and there are mature scales for measuring [13]. Finally, the theme of this guidelines adaptation was defined as distress.

The work plan of guidelines adaptation was written by the multidisciplinary panel of guidelines adaptation to guide the practice of this guidelines adaptation. Distress Management and Chinese Psychosocial Oncology Therapy Guidelines for Cancer Patients were included after searching, screening, assessment and selection, providing best evidence sources for the guidelines adaptation. Several clinical practice guidelines were excluded because they were not de novo clinical practice guidelines, such as A Pan Canadian Practice Guideline: Screening, Assessment and Care of Psychosocial Distress, Depression, and Anxiety in Adults with Cancer [26]. In this guidelines adaptation, AGREE II was used to assess the included guidelines' quality. The guidelines' currency was assessed by the publication date. The content was assessed in the screening. The acceptability and applicability of recommendations were assessed in the next step, because clinical context in the guidelines implementation site should be taken into consideration. The quality of the draft Cancer-related Distress Management Guidelines was high, and the feasibility, appropriateness, meaningfulness and effectiveness of 
most recommendations were good. However, the expression "spiritual and chaplaincy care" was impertinent in the social context of China. The guidelines adaptation panel discussed and analyzed the recommendations proposed by the stakeholders one by one, and revised the first draft guidelines to form the final version of Cancer-related Distress Management Guidelines.

This study has the following limitations. First, researchers only searched guidelines in English and Chinese. Some high quality guidelines in other languages may not be included. Second, only two de novo clinical practice guidelines were included in this study. Therefore, the included guidelines might not be fully representative of all guidelines for distress management worldwide.

\section{Conclusion}

The symptom burden of cancer patients in Shanghai was high, and the distress of physical symptoms and psychological symptoms were almost equivalent. Therefore, psychological symptoms and physical symptoms were both worthy of attention. Two clinical practice guidelines on distress management were included after searching, screening, evaluation and selection. The quality of the Distress Management Guidelines in Cancer Patients was high, and the feasibility, appropriateness, meaningfulness and effectiveness of most recommendations in the guidelines were good. The final guidelines were formed based on the revision of the first draft guidelines. Thus, guidelines adaptation was feasible in low and middle income countries based on CAN-IMPLEMENT and guided by the Knowledge-to-Action Framework.

\section{Additional File}

Additional file 1: The recommendations review form (FAME); Additional file 2: The results of guidelines' searching

\section{Abbreviations}

CANIMPLEMENT: A Guideline Adaptation and Implementation Planning Resource; AGREE II: Appraisal of Guidelines for Research and Evaluation II; QoL: Quality of Life; MSAS-SF: Memorial Symptom Assessment Scale Short Form; MSAS-SF-SC: Simplified Chinese Version of the Memorial Symptom Assessment Scale Short Form; NCCN: National Comprehensive Cancer Network

\section{Declarations}

\section{Acknowledgments}

The authors' special thanks go to Professor Margaret B. Harrison for her authorization of A Guideline Adaptation and Implementation Planning Resource (CAN-IMPLEMENT). The authors wish to thank master students from School of Nursing, Fudan University for the searching, screening, assessment, selection and drafting in the guidelines adaptation. The authors express their gratitude to the professors, administrators and staffs from Joanna Briggs Institute, Department of Psychological Medicine, Zhongshan Hospital Affiliated to Fudan University and Department of Oncology, Department of Social Work, Department of Integrated Therapy, Department of Nursing, Fudan University Shanghai Cancer Center for their participation in the peer review of the adapted guidelines and the routine distress management during the guidelines implementation. Finally, they wish to express their gratitude to all the patients who participated in this study.

\section{Funding}

This study was funded by Fudan-Fuxing Nursing Research Funds (No. FNF201701) from Fudan University, Shanghai, China. No conflicts of interest exist in the study. The sponsor did not participate in the study design, data collection, interpretation of results, or presentation of the information.

\section{Availability of data and materials}

The datasets used and/or analysed during the current study are available from the corresponding author on reasonable request.

\section{Authors' contributions}

YH and ZL mainly designed this research plan. $L F, Y Y, X Z$ and $Y W$ mainly completed the guidelines adaptation. LF, YY, XZ, MH, YL, FZ, $\mathrm{YW}$ and $\mathrm{ZH}$ are mainly responsible for the guidelines implementation. All authors read and approved the final manuscript. 
Ethical approval was obtained via School of Nursing Fudan University (IRB\#2017-9-3) and Fudan University Shanghai Cancer Centre (1710177-23).

\section{Consent for publication}

Not applicable.

\section{Competing Interests}

The authors declare they have no competing interests.

\section{References}

1. Siegel RL, Miller KD, Jemal A. Cancer statistics, 2020. CA Cancer J Clin. 2020;70:7-30.

2. Chen W, Zheng R, Baade PD, et al. Cancer statistics in China, 2015. CA Cancer J Clin. 2016;66:115-32.

3. Raphael D, Frey R, Gott M. Distress in post-treatment hematological cancer survivors: Prevalence and predictors. J Psychosoc Oncol. 2019:1-15.

4. Tantoy IY, Cooper BA, Dhruva A, et al. Changes in the Occurrence, Severity, and Distress of Symptoms in Patients With Gastrointestinal Cancers Receiving Chemotherapy. J Pain Symptom Manage. 2018;55:808-34.

5. Zhou T, Yang K, Thapa S, et al. Differences in Symptom Burden Among Cancer Patients With Different Stages of Cachexia. J Pain Symptom Manage. 2017;53:919-26.

6. Rohrl K, Guren MG, Astrup GL, et al. High symptom burden is associated with impaired quality of life in colorectal cancer patients during chemotherapy:A prospective longitudinal study. Eur J Oncol Nurs. 2019;44:101679.

7. Poort H, Jacobs JM, Pirl WF, et al. Fatigue in patients on oral targeted or chemotherapy for cancer and associations with anxiety, depression, and quality of life. Palliat Support Care. 2019:1-7.

8. Wang X, Lv Y, Li W, et al. Correlation between Psychosocial Distress and Quality of Life in Patients with Nasopharyngeal Carcinoma following Radiotherapy. J Oncol. 2018;2018:3625302.

9. Lee K, Oh EG, Kim S, et al. Symptom experiences and health-related quality of life among non-small cell lung cancer patients participating in clinical trials. J Clin Nurs. 2019;28:2111-23.

10. Fu L, Hu Y, Lu Z, et al. Validation of the Simplified Chinese Version of the Memorial Symptom Assessment Scale-Short Form Among Cancer Patients. J Pain Symptom Manage. 2018;56:113-21.

11. Chang VT, Hwang SS, Feuerman M, et al. The memorial symptom assessment scale short form (MSAS-SF). Cancer-Am Cancer Soc. 2000;89:1162-71.

12. Carlson LE, Zelinski EL, Toivonen Kl, et al. Prevalence of psychosocial distress in cancer patients across 55 North American cancer centers. J Psychosoc Oncol. 2019;37:5-21.

13. Holland JC, Deshields TL, Andersen B, et al NCCN Clinical Pratice Guidelines in Oncology: Distress Management. Version 2.2018. Available at: NCCN.org. Accessed March 312018.

14. Hong J, Wei Z, Wang W. Preoperative psychological distress, coping and quality of life in Chinese patients with newly diagnosed gastric cancer. J Clin Nurs. 2015;24:2439-47.

15. Stonelake-French H, Moos BE, Brueggen CM, et al. Understanding Distress in the Hospital: A Qualitative Study Examining Adults With Cancer. Oncol Nurs Forum. 2018;45:206-16.

16. Hedden L, Wassersug R, Mahovlich S, et al. Evaluating an educational intervention to alleviate distress amongst men with newly diagnosed prostate cancer and their partners. Bju Int. 2017;120:E21-9.

17. Pearson A, Wiechula R, Court A, et al. The JBI model of evidence-based healthcare. Int J Evid Based Healthc. 2005;3:207-15.

18. Jordan Z, Lockwood C, Munn Z, et al. Redeveloping the JBI Model of Evidence Based Healthcare. Int J Evid Based Healthc. 2018;16:227-41.

19. LOGAN JO, GRAHAM ID. Toward a Comprehensive Interdisciplinary Model of Health Care Research Use. Sci Commun. 1998;20:227-46. 
20. Kitson A, Harvey G, McCormack B. Enabling the implementation of evidence based practice: a conceptual framework. Qual Health Care. 1998;7:149-58.

21. Rycroft-Malone J, Kitson A, Harvey G, et al. Ingredients for change: revisiting a conceptual framework. Qual Saf Health Care. 2002;11:174-80.

22. Rycroft-Malone J, Harvey G, Seers K, et al. An exploration of the factors that influence the implementation of evidence into practice. J Clin Nurs. 2004;13:913-24.

23. Graham ID, Logan J, Harrison MB, et al. Lost in knowledge translation: time for a map? J Contin Educ Health Prof. 2006;26:13-24.

24. Harrison MB, van den Hoek J, for the Canadian Guideline Adaptation Study Group. CAN-IMPLEMENT: a guideline adaptation and implementation planning resource. Ontario: Queen's University School of Nursing and Canadian Partnership Against Cancer; 2012.

25. Harrison MB, Graham ID, van den Hoek J, et al. Guideline adaptation and implementation planning: a prospective observational study. Implement Sci. 2013;8:49.

26. Howell D, Keshavarz H, Esplen MJ, Hack T, Hamel M, Howes J, Jones J, Li M, Manii D, McLeod D, Mayer C, Sellick S, Riahizadeh S, Noroozi H, Ali, M on behalf of the Cancer Journey Advisory Group of the Canadian Partnership Against Cancer. A Pan Canadian Practice Guideline: Screening, Assessment and Care of Psychosocial Distress, Depression, and Anxiety in Adults with Cancer. Toronto: Canadian Partnership Against Cancer and the Canadian Association of Psychosocial Oncology; 2015.

27. Tang LL. Chinese Psychosocial Oncology Therapy Guidelines for Cancer Patients. Beijing: People's Medical Publishing House; 2016.

\section{Figures}




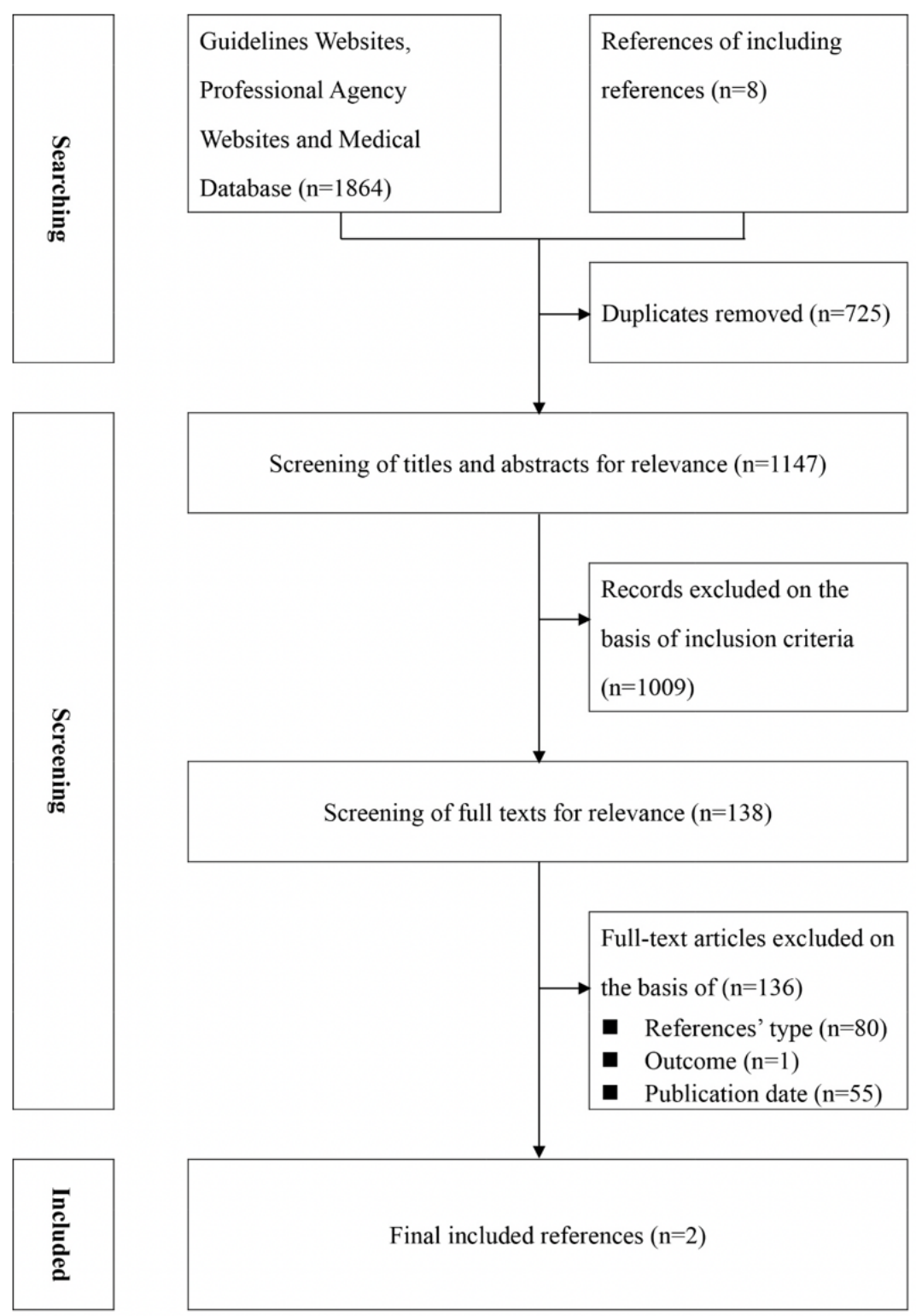

\section{Figure 1}

The process of guidelines' searching and screening

\section{Supplementary Files}

This is a list of supplementary files associated with this preprint. Click to download.

- AdditionalfilesAdaptationPart.docx

- AdditionalfilesAdaptationPart.docx

- AdditionalfilesAdaptationPart.docx 